\title{
Una vía de emprendimiento en los estudiantes del Tecnológico Espíritu Santo (TES)
}

\section{A path to entrepreneurship for students of the Tecnológico Espíritu Santo (TES)}

Lic. Martha María Fernández Rodríguez. PhD

Instituto Superior Tecnológico Espíritu Santo, Ecuador

Lcda. Sheylah Hoppe Coronel, MBA

Instituto Superior Tecnológico Espíritu Santo, Ecuador

Autor para correspondencia: mmfernandez@tes.edu.ec, shoppe@tes.edu.ec

Fecha de recepción: 2 de enero de 2018 - Fecha de aceptación: 2 de agosto de 2018

Resumen: Se presentaron los resultados de la aplicación de los proyectos de asignaturas en el Tecnológico Espíritu Santo (TES) como una vía de emprendimiento en los estudiantes. El objetivo de este trabajo fue demostrar en la práctica cómo los proyectos de asignaturas constituyen una metodología que caracteriza a la institución y que permite preparar a los estudiantes para emprender acorde a la especialidad que estudien. El problema científico que motivó la realización de esta investigación fue: ¿Cómo los proyectos de asignatura contribuyen a los Emprendimientos en el TES? El contenido del artículo formó parte de los resultados de la puesta en práctica de esta metodología que caracteriza la docencia teórica-práctica en la institución. Se emplearon métodos empíricos como la encuesta, auxiliados de técnicas como la revisión de documentos. Se logró como resultado demostrar a través de uno de los grandes eventos que se realizan en la institución "El Open House" los Emprendimientos que presentan los estudiantes en los que se demuestran competencias y habilidades propias de la profesión en la que se graduarán.

Palabras Claves: proyectos de asignaturas; emprendimientos; competencias profesionales

Abstract: The results of the application of the subject projects in the Technologic Espiritu Santo were presented as a way of entrepreneurship in the students. The objective of this work was to demonstrate in practice how the subject projects constitute a methodology that characterizes the institution and that allows preparing students to undertake according to the specialty they study. The scientific problem that motivated the realization of this investigation was: How the subject projects contribute to the entrepreneurship in the TES? The content of the article was part of the results of the implementation of this methodology that characterizes the theoretical-practical teaching in the institution. Empirical methods such as the survey were used, assisted by techniques such as the review of documents. It was achieved as a result to demonstrate through one of the great events that take place in the institution "The Open House" the Entrepreneurship that the students present in which they demonstrate competences and abilities of the profession in which they will graduate.

Key words: projects of subjects; entrepreneurship; professional skills 


\section{Introducción}

La metodología de aprendizaje supone buscar respuestas al cómo enseñar, es decir la estructura de las actividades de enseñanza-aprendizaje en la que van a participar los alumnos con el fin de alcanzar los objetivos propuestos con los contenidos seleccionados, mediante una acción intencional, sistemática y planificada.

El TES tiene entre su metodología de aprendizaje los "Proyecto de asignatura". Los mismos constituyen la modalidad investigativa que permite a los estudiantes adquirir un mayor dominio y actualización de los métodos y técnicas de investigación científica características de la profesión y que propicia la generalización y aplicación de los contenidos de la carrera para la solución de un problema de la práctica profesional.

Para ello, el estudiante debe someterse a un proceso investigativo que permita la integración de los contenidos académicos con la problemática de la profesión y la futura vida laboral en el campo de estudio.

Los temas para la realización del proyecto de asignatura serán formulados por los estudiantes y aprobados por los profesores. Los mismos deben estar relacionados con la carrera y la materia, que sea de interés y contenido científico, que vaya dirigido fundamentalmente a resolver un problema de la práctica profesional y con las líneas de investigación institucional.

Esta metodología facilita un aprendizaje teórico-práctico conducente al desarrollo de competencias profesionales capaces de expresarse en los proyectos de asignaturas que se presentan al finalizar cada período académico. Los proyectos de asignaturas se presentan en eventos a nivel Institucional como Open House y La Feria Gastronómica.

Desde esta perspectiva el problema científico que motivó la realización de esta investigación es: ¿Cómo los proyectos de asignatura contribuyen a los Emprendimientos en el TES? Y con ello, se pretende como objetivo demostrar en la práctica cómo los proyectos de asignaturas constituyen una metodología que caracteriza a la institución y que permite preparar a los estudiantes para emprender acorde a la especialidad que estudien.

\section{Fundamentos teóricos de los Proyectos de Asignatura como vía de emprendimiento}

Dentro de las corrientes pedagógicas se encuentra el Constructivismo. La misma fue creada por Ernest Van Glasersed, basándose en los conocimientos constructivistas que señalan la necesidad de otorgar al estudiante herramientas que propicien resolver una situación problemática lo cual facilita que sea un ente activo del proceso.

Dentro de los representantes destacados del constructivismo cabe destacar a Jean Peaget y Leo Vygotski. El primero se concentra en la construcción del conocimiento desde la interacción con el medio mientras Vygotski se centra en cómo el medio social permite una reconstrucción interna. 
Desde esta perspectiva teórica se asume en el TES un Modelo Constructivista, que contribuye a la indagación, reflexión y desafíos que se concreten en proyectos de asignaturas que tributa a una enseñanza teórico-práctica y con la participación y colaboración de todos, garantizando un clima de confianza y comunicación en el proceso educativo.

Y en este sentido, el proceso de enseñanza-aprendizaje se desarrolla desde contenidos teóricos y conceptuales que guíen una estrategia de aprendizaje en la que se le ofrezca al estudiante la oportunidad de adquirir conocimiento y practicarlo en el propio escenario del contexto de su materia. En este mismo orden de ideas los estudiantes interactúan en la construcción del conocimiento y se producen relaciones de cooperación y colaboración.

Ello conlleva a considerar profesores que:

- Contribuyan al desarrollo de la autonomía en los estudiantes.

- Contribuyan al desarrollo de habilidades y competencias que se correspondan con la misión y visión institucional y de la carrera.

- Desarrollen habilidades investigativas.

- Desarrollen una enseñanza que se concrete en proyectos de asignaturas.

- Eduquen en el desarrollo de emprendimientos.

Desde esta mirada, los proyectos de asignaturas contribuirán a ir formando en los estudiantes habilidades investigativas que le permiten validar en la práctica profesional y ayudar a solucionar falencias existentes a la vez que le facilita elaborar proyectos de emprendimientos acorde a su perfil.

Ello propicia una combinación de enseñanza y emprendimiento en la formación profesional de los estudiantes. Concordando con Solis (2002) quien señala:

Si la educación es el medio por el cual logro modificar actitudes y comportamientos, casi diría que hay una relación absoluta entre educación y entrepreneurship, vale la pena, entonces pensar en educar a los emprendedores. Puede ser que haya muchos emprendedores que nazcan, pero seguro que todos necesitan hacerse y capacitarse como tales. (Carbonell, 2002).

Siendo consecuente con el modelo constructivista del TES y la concepción de Solis 2002, si la educación contribuye a formar a los estudiantes, la aspiración es que estos se conviertan en protagonistas de cambiar el contexto donde se desenvuelven en su formación profesional, de ahí, que desde la metodología a emplear en el TES los proyectos de asignaturas contribuirán a ayudar a formar estudiantes y educar a los emprendedores.

Del mismo modo:

...la educación en entrepreneurship (...) puede forjar actitudes emprendedoras en ellos. Puede promover cualidades psicológicas favorables para la actividad emprendedora, tales como la autoconfianza, la autoestima, la auto eficacia y la necesidad de logro. Inclusive, la educación entrepreneurship para los jóvenes, puede colaborar en evitar la generación de actitudes socialmente no deseables, como la vagancia o la delincuencia. (Formichella, 2002) 
En ese sentido, Stevenson (2000), plantea: “el entorno es importante, y es más factible que un individuo pueda comenzar a tener actitudes emprendedoras si actúa en un contexto en el que se facilita el reconocimiento de la oportunidad y su persecución”. (Formichella, 2002)

El Instituto Técnico Superior Espíritu Santo, desarrolla una docencia investigativa como parte de los procesos de aprendizaje teórico-práctico de la carrera. La misma se guía por los tipos de investigación que se asumen estos son: exploratoria, diagnóstica y aplicada. Estas se proyectan en los diferentes espacios de aprendizajes como son la clase, la práctica pre profesional, el trabajo de titulación, eventos de emprendimientos, teórico-prácticos y proyectos de vinculación con la sociedad.

Las estrategias de investigación para el aprendizaje, que se implementa por las carreras, para desarrollar el aprendizaje teórico práctico, serán las encargadas de guiar, ayudar y establecer el modo de aprender. En las técnicas de estudio usadas por cada estudiante y su desarrollo en la práctica pre profesional, aplicarán las estrategias de investigación mediante procedimientos concretos para cada una que conducirán a la elaboración de proyectos de asignaturas que tributen al emprendimiento.

Estas deben completarse de forma lo más individual posible, para ajustarnos al caso de cada estudiante. Son procedimientos que incluyen técnicas, operaciones y actividades que buscan el "aprender a aprender". Estas estrategias están articuladas a las líneas de investigación institucional y las problemáticas del contexto de la profesión que se resuelven dentro de las cuales están determinadas en la carrea de Marketing y Comunicación, objeto de esta investigación las siguientes:

Tabla 1. Líneas de investigación y problemática a resolver

\begin{tabular}{|c|c|c|c|}
\hline$\#$ & Carrera & $\begin{array}{c}\text { Líneas de } \\
\text { investigación }\end{array}$ & Problemáticas a resolver \\
\hline 1 & $\begin{array}{l}\text { Tecnología Superior } \\
\text { Comunicaciones y } \\
\text { Relaciones Públicas }\end{array}$ & $\begin{array}{l}\text { Línea } 1 . \\
\text { Comunicación } \\
\text { tecnológica } \\
\text { Línea } 5 . \\
\text { Marketing, } \\
\text { tecnología y } \\
\text { comunicación }\end{array}$ & $\begin{array}{l}\text { Se ejecutan campañas comunicacionales por personas } \\
\text { sin los conocimientos teóricos básicos que limita } \\
\text { objetividad y trasparencia de los mensajes } \\
\text { No siempre, se aplican las herramientas de } \\
\text { investigación con la profundidad requerida lo cual } \\
\text { impide conocer las necesidades de los públicos y del } \\
\text { mercado lo que limita la proyección de las empresas. } \\
\text { No siempre, se realizan los estudios pertinentes que } \\
\text { sirvan para mejorar la calidad de la comunicación y la } \\
\text { relación con los prospectos, ni se consideran sus } \\
\text { necesidades para conseguir cambios en la organización } \\
\text { centradas en las relaciones de mutuo beneficio. } \\
\text { Falta de estrategias comunicacionales profesionales, en } \\
\text { los cuales se vean verdaderamente dirigida la } \\
\text { comunicación efectiva y una gestión humana y } \\
\text { empática con los involucrados. } \\
\text { Limitado personal preparado para promover el } \\
\text { desarrollo de industrias culturales y creativas que } \\
\text { permitan desarrollar campañas publicitarias y de } \\
\text { comunicación. } \\
\text { Con frecuencia, se realizan emprendimientos con } \\
\text { insuficiente innovación tecnológica y poco uso de la } \\
\text { tecnología y mina de información, además de poca }\end{array}$ \\
\hline
\end{tabular}


2 Tecnología Superior Marketing

3 Tecnología Superior Publicidad
Línea 2.

Competitividad

Global.

Línea 5.

Marketing, tecnología y comunicación

Línea 1.

Comunicación tecnológica

Línea 5.

Marketing, tecnología y comunicación Línea 6. Publicidad, arte y diseño retroalimentación de expertis comercial, lo cual no permite análisis probabilísticos para bajar el riesgo y conocer el retorno de las inversiones.

Se crean empresas sobre la base de la empírea, sin un proceso de investigación profunda que conlleve a la falta de permanencia en el mercado.

Se realizan proyectos sin tener en consideración el impacto social, el contexto socioeconómico y la asertividad en las decisiones para proyectarse a largo plazo

Limitado personal preparado para detectar oportunidades de negocios, en el contexto local, nacional, regional y mundial, lo que limita la competitividad de la empresa.

Con frecuencia, se realizan emprendimientos con insuficiente innovación tecnológica y poco uso de la tecnología y mina de información, lo cual no permite análisis probabilísticos para bajar el riesgo y conocer el retorno de las inversiones.

Se ejecutan campañas comunicacionales por personas sin los conocimientos teóricos básicos que no permiten maximizar el ROI de las empresas.

Con frecuencia se realizan programas y proyectos publicitarios que no se ajustan al contexto socioeconómico del país y no se ajusta a la normativa legal existente

No siempre se establecen los análisis de precios con las herramientas del marketing necesarios, ni se analizan adecuadamente los canales de distribución y puntos de ventas, lo que conlleva a elevar el riesgo de fracaso del producto en el mercado.

Con frecuencia, se realizan emprendimientos con insuficiente innovación tecnológica y poco uso de la tecnología y mina de información, además de poca retroalimentación de expertis comercial, lo cual no permite análisis probabilísticos para bajar el riesgo y conocer el retorno de las inversiones.

Falta de procesos creativos profesionales, en los cuales se vean verdaderamente dirigida la comunicación visual con una conceptualización y mensaje creativo

Falta de planificación en la comunicación, campañas poco creativas y de bajo impacto para el cliente objetivo, poco conocimiento del mercado, del consumidor y de como este se relaciona con las marcas a través de los medios de comunicación, lo que permite inferir la necesidad de profesionales en la rama que estén preparados para asumir una diversidad de roles y funciones asociadas a los procesos básicos de la profesión.

4 Tecnología Superior Línea2. Marketing Tecnología Competitividad Superior Comunicaciones y Global. Relaciones Públicas Línea 5. Tecnología Superior Publicidad Tecnología Marketing, tecnología y comunicación
Se ejecutan ventas por personas sin los conocimientos teóricos básicos que no permiten maximizar la eficiencia de las ventas.

La comunicación y proyección por parte de los vendedores no es eficiente porque carecen de conocimientos informáticos y de expresión oral y 
escrita que no ayudan a generar la confianza del consumidor.

Muchos proyectos de ventas o de otra índole no están correctamente contextualizados y no consideran aspectos éticos.

No siempre, se realizan los estudios pertinentes que sirvan para mejorar la calidad del servicio y la relación con los prospectos, ni se consideran sus necesidades para conseguir cambios en la organización centrada en el cliente.

No siempre, se establecen los análisis de precios con las herramientas del marketing que complementan las técnicas de ventas, ni se analizan adecuadamente los canales de distribución y puntos de ventas, lo que conlleva a elevar el riesgo de fracaso del producto en el mercado.

Separación entre la práctica del marketing y las ventas per se, lo cual deteriora la fidelización por parte de los clientes

Vendedores con falta de preparación en las últimas técnicas de negociación que no permiten realizar acuerdos que generen ingresos para una empresa

Con frecuencia, se realizan emprendimientos con insuficiente innovación tecnológica y poco uso de la tecnología y mina de información, además de poca retroalimentación de expertis comercial, lo cual no permite análisis probabilísticos para bajar el riesgo y conocer el retorno de las inversiones.

5 Producción en Comunicación Línea 1. Audiovisual con nivel equivalente a Técnico Superior
Comunicación tecnológica

Línea 6. Publicidad, arte y diseño
Se realiza el ejercicio de la profesión relacionada al manejo de la comunicación audiovisual, por personal empírico que carece de la preparación académica necesaria, lo que incide en la falta de sentido ético.

Se realizan proyectos tecnológicos sin los softwares adecuados para su creación, por falta de personal capacitado, lo que conlleva a perdida de eficiencia del trabajo.

Se desarrollan emprendimientos en el sector de las comunicaciones audiovisuales que no responden a las necesidades del país

Se realizan producciones en cine, televisión, radio y multimedia, sin el uso de guiones o sin la estructura adecuada, que conlleva a la improvisación y falta de calidad en los resultados del producto.

Mal manejo de la narrativa audiovisual, productores no consideran la naturaleza del medio a través del cual se transmite un mensaje.

El desconocimiento de las herramientas de iluminación y de escenografía conlleva a realizar producciones audiovisuales de mala calidad, sin considerar la importancia del manejo previo de la imagen.

Ineficiente adaptación a los cambios que limita su aprovechamiento para conseguir resultados de impacto en materia audiovisual, lo que no permite competir con el mercado internacional.

Fuente: Modelo de Vinculación con la sociedad del TES (Tecnológico Espíritu Santo, 2017) 


\section{Metodología}

\section{Materiales y métodos}

El estudio se realizó en el Instituto Técnico Superior Espíritu Santo (TES) de la ciudad de Guayaquil, Ecuador y abarcó las carreras del área de Marketing y Comunicación: Tecnología Superior Marketing, Tecnología Superior Comunicaciones y Relaciones Públicas, Tecnología Superior en Publicidad, Tecnología Superior en Ventas y Producción en Comunicación Audiovisual con nivel equivalente a Técnico Superior.

La Población objeto de estudio son 125 estudiantes de estas carreras que cursan materias en el primer período académico del 2018, las mismas se presentaron en el evento Hopen House, de ello se seleccionó de manera aleatoria una muestra del 100 estudiantes que representan el $80 \%$ del total de la población. Para realizar la investigación se emplean métodos del nivel empírico: como la entrevista y técnicas como la guía de revisión de documentos.

La entrevista se emplea para obtener información relacionada con la elaboración de proyectos de asignaturas en el semestre 2018 y cómo los mismos contribuyen a algún tipo de emprendimiento en el campo objeto de estudio. Guía de revisión de documentos: Se emplea para revisar los proyectos de asignaturas presentados por los estudiantes y comprobar cuántos están en la línea de innovación y emprendimiento en el campo objeto de estudio.

\section{Resultados y discusión}

La carreras del área de Marketing y Comunicación tiene como tradición realizar el evento Hopen House en la que los estudiantes exponen sus proyectos de asignaturas y evidencian los conocimientos y las competencias adquiridas en las asignaturas recibidas en el período académico en coordinación con aprendizajes que le han antecedido. Cada año se selecciona una temática que es propuesta por el estudiante y los obliga a investigar sobre la misma para poder representarla en el orden cultural y estético al evento. En este año 2018 fue la temática de Películas.

Para corroborar si los proyectos de asignaturas contribuyen al desarrollo de habilidades investigativas y competencias profesionales que tributen al emprendimiento se realizó una encuesta.

\section{Resultado de la encuesta.}




\section{¿Aplican en las materias de la carrera como metodología el} proyecto de asignatura?

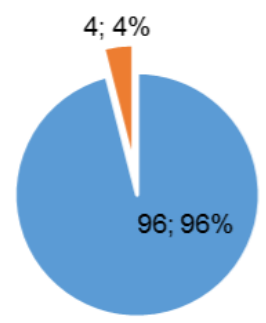

$$
\text { -SI }=\mathrm{NO}
$$

Figura 1. Metodología de proyectos de asignatura Fuente. Encuesta

De los resultados se confirma que $96 \%$ de las asignaturas de la carrera utilizan la metodología de proyectos, siendo consecuente con la metodología constructivista que se aplica en el modelo institucional.

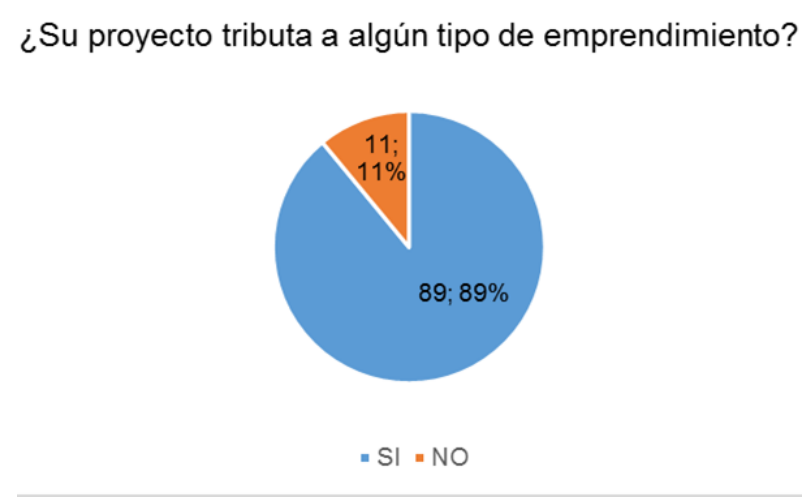

Figura 2. Proyecto tributa a un emprendimiento

Fuente. Encuesta

La mayoría representada por el $89 \%$ manifiesta que los proyectos tributan a algún tipo de emprendimiento, corroborándose que se trabaja desde el inicio del período académico en función de los estudiantes terminen la materia presentando un proyecto que responda al campo de la profesión y contribuya a la demostración de las competencias adquiridas. 
Considera que los proyectos de asignaturas permiten:

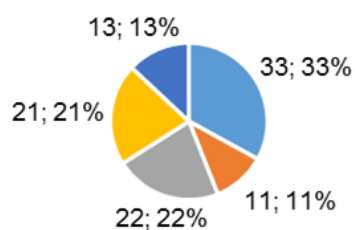

- Identificar competencias, a partir del uso de recursos tecnológicos

- Promover aprendizajes autónomo

- Dominar los aspectos teóricos y metodológicos de la investigación teórica

- Desarrollar propuestas de emprendimiento en el campo de la profesión

- Desarrollar habilidades investigativas

Figura 3. Contribución de los proyectos de asignaturas a la formación profesional Fuente. Encuesta

Los encuestados refieren (67 \%) cómo los proyectos contribuyen a su formación profesional, desglosado en un 33,3\% identificar competencias a partir del uso de recursos tecnológicos, que los proyectos de asignaturas contribuyen al desarrollo de habilidades investigativas $13 \%$, y a desarrollar propuestas de emprendimiento en el campo de la profesión $21 \%$, de ello se infiere que la metodología de desarrollo de proyecto influye en su formación profesional y los prepara para el desempeño laboral.

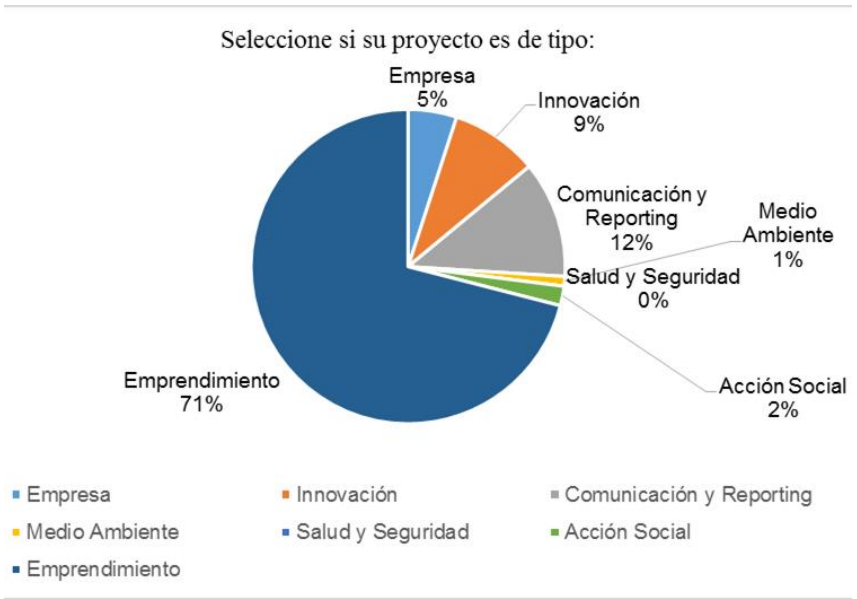

Figura 4. Tipo de proyecto

Fuente. Encuesta

Como se observa en los resultados ante la selección del tipo de proyecto el 71, \% que representa la mayoría presentan proyectos de emprendimientos, sin descartar que existen otras opciones como empresa e innovación que también fueron seleccionadas por los encuestados y que también tributan al emprendimiento, muestra de que se realizan emprendimientos propios del campo de la profesión.

En la revisión de los proyectos de asignaturas al finalizar el período académico que se presentaron al evento Hopen House se aprecia los siguientes resultados. 
Tabla 2. Relación de proyectos en Hopen House 2018. TES

\begin{tabular}{|c|c|c|c|}
\hline \# & TITULO DEL PROYECTO & MATERIA & $\begin{array}{c}\text { TIPO DE } \\
\text { PROYECTO }\end{array}$ \\
\hline 1 & $\begin{array}{l}\text { Estimulación de la comunicación como medio para la } \\
\text { formación cultural de la población ecuatoriana }\end{array}$ & Teoría de la comunicación & $\begin{array}{l}\text { Comunicación } \\
\text { y reporting }\end{array}$ \\
\hline 2 & $\begin{array}{l}\text { Desarrollo de la comunicación asertiva en el aprendizaje de } \\
\text { los alumnos del TES }\end{array}$ & Teoría de la comunicación & $\begin{array}{l}\text { Comunicación } \\
\text { y reporting }\end{array}$ \\
\hline 3 & $\begin{array}{l}\text { Influencia de la comunicación educativa en los resultados } \\
\text { del fútbol ecuatoriano }\end{array}$ & Teoría de la comunicación & $\begin{array}{l}\text { Comunicación } \\
\text { y reporting }\end{array}$ \\
\hline 4 & Importancia de la comunicación en la relación padre e hijo & Teoría de la comunicación & $\begin{array}{l}\text { Emprendimient } \\
\mathrm{o}\end{array}$ \\
\hline 5 & Crema ACNELLA & Gerencia de marketing & $\begin{array}{l}\text { Emprendimient } \\
\mathrm{o}\end{array}$ \\
\hline 6 & Brownchi (brownie de machica) & Fundamentos de marketing & $\begin{array}{l}\text { Emprendimient } \\
\mathrm{o}\end{array}$ \\
\hline 7 & Mourinaca & Fundamentos de marketing & $\begin{array}{l}\text { Emprendimient } \\
\text { o }\end{array}$ \\
\hline 8 & Cheeseroll a lo macho & Fundamentos de marketing & $\begin{array}{l}\text { Emprendimien } \\
\text { to }\end{array}$ \\
\hline 9 & Barra de chocolate vinótica & Fundamentos de marketing & Innovación \\
\hline $\begin{array}{l}1 \\
0\end{array}$ & Lunacai & Fundamentos de marketing & $\begin{array}{l}\text { Emprendimien } \\
\text { to }\end{array}$ \\
\hline $\begin{array}{l}1 \\
1\end{array}$ & MorinCrunch & Fundamentos de marketing & Innovación \\
\hline $\begin{array}{l}1 \\
2\end{array}$ & Escuela de formación de vendedores & Administración de ventas & Empresa \\
\hline $\begin{array}{l}1 \\
3\end{array}$ & $80 ` \mathrm{~S}$ forever & Marketing de servicios & Innovación \\
\hline $\begin{array}{l}1 \\
4\end{array}$ & Servicio de entretenimiento & Marketing de servicios & $\begin{array}{l}\text { Entretenimient } \\
\text { o familiar }\end{array}$ \\
\hline $\begin{array}{l}1 \\
5\end{array}$ & Cinemasmart & Campaña de publicidad & Innovación \\
\hline $\begin{array}{l}1 \\
6\end{array}$ & Ice Colors & Campaña de publicidad & Innovación \\
\hline $\begin{array}{l}1 \\
7\end{array}$ & Coffee garden & Fundamentos de publicidad & Innovación \\
\hline $\begin{array}{l}1 \\
8\end{array}$ & Pizzaarepa dulce & Fundamentos de publicidad & Innovación \\
\hline $\begin{array}{l}1 \\
9\end{array}$ & Torta vegana & Estrategia publicitaria & Innovación \\
\hline $\begin{array}{l}2 \\
0\end{array}$ & Mermeladas light & Estrategia publicitaria & Innovación \\
\hline $\begin{array}{l}2 \\
1\end{array}$ & La gala & Pensamiento crítico I y II & $\begin{array}{l}\text { Emprendimien } \\
\text { to }\end{array}$ \\
\hline $\begin{array}{l}2 \\
2\end{array}$ & Granizado con ingredientes auténticos del Ecuador & $\begin{array}{l}\text { Metodología de la } \\
\text { investigación }\end{array}$ & Innovación \\
\hline $\begin{array}{l}2 \\
3\end{array}$ & Mana do Ceu & Fundamentos de Ventas & Innovación \\
\hline $\begin{array}{l}2 \\
4\end{array}$ & Back to italianisimo - VESPA & Fundamentos de Ventas & $\begin{array}{l}\text { Plan } \\
\text { financiamiento }\end{array}$ \\
\hline $\begin{array}{l}2 \\
5\end{array}$ & ITV & Fundamentos de Ventas & $\begin{array}{l}\text { Emprendimien } \\
\text { to }\end{array}$ \\
\hline $\begin{array}{l}2 \\
6 \\
\end{array}$ & Revista Vanguardista & $\begin{array}{l}\text { Producción de medios } \\
\text { impresos I }\end{array}$ & $\begin{array}{l}\text { Comunicación } \\
\text { y reporting }\end{array}$ \\
\hline
\end{tabular}




\begin{tabular}{|c|c|c|c|}
\hline 2 & Tea Bubblymilk & Fundamentos de marketing & Emprendimient \\
\hline $\begin{array}{l}7 \\
2\end{array}$ & \multirow{2}{*}{ Vicios de Lenguaje } & Lenguaje y Comunicación & $\begin{array}{l}\text { O } \\
\text { Emprendimient }\end{array}$ \\
\hline 8 & & & $\mathrm{O}$ \\
\hline 2 & \multirow[t]{2}{*}{ Estrategias y Oportunidades } & Expresión Oral y Escrita & Emprendimient \\
\hline 9 & & & o \\
\hline 3 & \multirow[t]{2}{*}{ Técnicas de Lenguaje } & Expresión Oral y Escrita & Emprendimient \\
\hline 0 & & & o \\
\hline 3 & \multirow[t]{2}{*}{ Plan Lector } & Expresión Oral y Escrita & Emprendimient \\
\hline 1 & & & o \\
\hline 3 & \multirow[t]{2}{*}{ Técnicas de expresión Oral } & Expresión Oral y Escrita & Emprendimient \\
\hline 2 & & & o \\
\hline 3 & \multirow[t]{2}{*}{ Pensamiento Divergente y Convergente } & Pensamiento Crítico I & Emprendimient \\
\hline 3 & & & o \\
\hline 3 & \multirow[t]{2}{*}{ Plan Lector } & Pensamiento Crítico I & Emprendimient \\
\hline 4 & & & o \\
\hline 3 & \multirow[t]{2}{*}{ Show Musical } & Psicología de la & Emprendimient \\
\hline 5 & & Comunicación & o \\
\hline 3 & \multirow[t]{2}{*}{ Show Musical } & Psicología de la & Emprendimient \\
\hline 6 & & Comunicación & o \\
\hline 3 & \multirow[t]{2}{*}{ Show Musical } & Psicología de la & Emprendimient \\
\hline 7 & & Comunicación & o \\
\hline 3 & \multirow[t]{2}{*}{ Pantuflas con linterna } & Fundamentos de marketing & Emprendimient \\
\hline 8 & & & o \\
\hline 3 & \multirow[t]{2}{*}{ Negociando con IT } & Manejo de Crisis y Teoría & Emprendimient \\
\hline 9 & & de la comunicación & o \\
\hline 4 & \multirow[t]{2}{*}{ Medios de difusión impreso y digitales para empresas } & Redacción y géneros & Emprendimient \\
\hline 0 & & periodísticos & $\mathrm{o}$ \\
\hline
\end{tabular}

\section{Discusión}

Del análisis de los resultados se infieren que los proyectos de asignaturas contribuyen a:

- Identificar competencias, a partir del uso de recursos tecnológicos y del desarrollo actitudinal para la comprensión de situaciones complejas desarrolladas en el campo de las profesiones, ajustada a las tendencias internacionales, nacionales y locales donde los estudiantes deben detectarlas y ajustar sus emprendimientos a partir de los resultados investigados.

- Desarrollar aprendizaje que concluyen al finalizar cada período académico como resultado con un proyecto de asignatura, que es una estrategia fundamentada en un enfoque constructivista, que promueve el aprendizaje autónomo y hace que cobre relevancia para el estudiante el papel de investigador y de emprendimiento.

- Dominio de los aspectos teóricos y metodológicos de la investigación exploratoria, teórica y aplicada de acuerdo con las áreas de las carreas.

- Desarrollo de actitudes de liderazgo, colaboración, innovación, investigación y emprendimiento en la profesión. 
- Desarrollo de propuestas de investigación en el campo de la profesión, donde pudiesen demostrar sus habilidades para trabajar en equipo.

- Conocimiento y uso de los métodos y técnicas de investigación para su aplicación en el campo de estudio.

- Desarrollo de proyectos de asignaturas de tipo de emprendimiento que favorece su formación profesional.

\section{Conclusiones}

Se puede concluir que la metodología de desarrollo de Proyectos de asignaturas que se aplica en el TES, contribuye al desarrollo de habilidades investigativas, al mismo tiempo que desarrolla competencias profesionales que ayudan a la formación de emprendedores, lo que facilitará su desempeño profesional. Los proyectos se asignaturas que estén en la línea de reformar el patrón de producción al explotar una innovación o modificar nuevos productos o introducir nuevos fuente de insumos que ayudan a mejorar la calidad del sector donde se desarrolla pueden considerarse como proyectos de asignaturas que tributen a emprendimiento. Ello es demostrativo de la formación de los profesionales de calidad que facilitará el desempeño laboral en el sector donde se está formando.

\section{Agradecimiento}

A todos los estudiantes y profesores de las carreras del área de Marketing y Comunicación del TES, que contribuyeron con la colaboración aportando información para lograr esta investigación.

\section{Bibliografía}

Bernal, C. (2010). Metodología de la investigación. Colombia, Pearson.

Bunge, M. (2007). A la Caza de la Realidad. Barcelona España. Editorial Gedesa.

Bunge Mario. (2007). La ciencia. Su método y su filosofía. Obtenido de: http://jmvertiz.posgrado.unam.mx/pmdcmos02/formatos/laciencia.pdf

Carbonell, S. S. (2002). V Encuentro de Empresarios, organizado por Asociación Cristiana de Dirigentes de Empresas. Obtenido de www.redalyc.org/html/849/84917310058/

Carretero, M. (1993). Constructivismo e Educación. Aique. Bs As. Federación de educadores Bonaerenses.

Duarte Tito Ruiz, Tibana, Miriam. (2009). Emprendimiento: una opción para el desarrollo. Año XV, No. 43. Universidad Tecnológica de Pereira. Colombia. Scientia et technica. 
Formichella, M. (2002). El concepto de emprendimiento y su relación con la educación, el empleo y el desarrollo local,. Buenos Aires: Monografía Tres Arroyos. Obtenido de https://www.calameo.com/books/003321091ebda840288b8

Hernández Sampieri, Roberto, Carlos Fernández Collado y Pilar Baptista Lucio. (2002). Metodología de la Investigación. México, Mc Graw Hill.

Méndez Rafael.(2008). Formulación y evaluación de proyectos, enfoque para emprendedores. Icontec Internacional, 5ta edición Bogotá.

Pozo, Juan Ignacio. (2006). Teoría Cognitiva del aprendizaje. Madrid. Editorial Morata SL.

Tecnológico Espíritu Santo. (2017). Modelo de Vinculación con la Sociedad.Documento Inédito de la Institución.

Schumeter, Joseph Alois. (2015). Capitalismo Socialismo y democracia. Traducción de José Diaz García y Alejandro Limeres. Volumen I. Barcelona.

Santiuste Bermejo Víctor. Cuadernos de Educación aproximación al concepto de aprendizaje contructivista.. Obtenido de http/:www.indenet. Santillana. es/res/archivos/infantil cuadernos/const 1

Selltiz Claire. (1980). Método de Investigación en las Ciencias Sociales. Obtenido de https://www.urbe.edu/UDWLibrary/InfoBook.do?id=2843

Shunk Sale. H. (2012). Teoría del aprendizaje una perspectiva educativa. México. Editorial Pearson Educación. 